\title{
The interplay between cell signaling and the mevalonate pathway in cancer
}

\author{
Peter J. Mullen, Rosemary Yu, Joseph Longo, Michael C. Archer, \\ and Linda Z. Penn
}

Version Post-print/accepted manuscript

Citation Mullen, Peter J., et al. "The interplay between cell signalling and the

(published version) mevalonate pathway in cancer." Nature Reviews Cancer 16.11 (2016):

718. doi: $10.1038 /$ nrc. 2016.76

\section{How to cite TSpace items}

Always cite the published version, so the author(s) will receive recognition through services that track citation counts, e.g. Scopus. If you need to cite the page number of the author manuscript from TSpace because you cannot access the published version, then cite the TSpace version in addition to the published version using the permanent URI (handle) found on the record page.

This article was made openly accessible by $U$ of $T$ Faculty. Please tell us how this access benefits you. Your story matters. 


\section{The interplay between cell signaling and the mevalonate pathway in cancer}

2 Peter J Mullen ${ }^{1 *}$, Rosemary $\mathrm{Yu}^{1,3 *}$, Joseph Longo ${ }^{1,3 *}$, Michael C Archer ${ }^{2}$, Linda Z Penn ${ }^{1,3}$

$3 \quad{ }^{1}$ Princess Margaret Cancer Centre, University Health Network, Toronto, Ontario, Canada.

$4{ }^{2}$ Department of Nutritional Sciences, Faculty of Medicine, University of Toronto, Toronto, Ontario,

5 Canada.

$6{ }^{3}$ Department of Medical Biophysics, Faculty of Medicine, University of Toronto, Toronto, Ontario,

7 Canada.

$8 *$ contributed equally

\section{Biography}

10 LZP focuses on two major areas of research: 1) understanding the regulation and function of the Myc

11 oncogene; and 2) investigating the role of the mevalonate pathway in tumorigenesis, and how best to use

12 statins to target this cancer vulnerability and impact cancer patient care. MCA is now Professor Emeritus

13 and his research previously focused on the role of environmental factors and susceptibility genes in

14 cancer development. Research trainees in the Penn lab include PJM (Post-doctoral Fellow), RY and JL

15 (PhD students).

16 Abstract

17 The mevalonate pathway is an essential metabolic pathway that uses acetyl-CoA to produce sterols and

18 isoprenoids integral to tumour growth and progression. In recent years, many oncogenic signaling

19 pathways have been shown to increase the activity and/or expression of mevalonate pathway enzymes.

20 This review summarizes recent advances and discusses the unique opportunities to immediately target this

21 metabolic vulnerability with approved agents, such as the statin family of drugs, to impact patient care

22 and outcome. 


\section{Key points}

2 1. Mevalonate pathway metabolites are essential for cancer cell survival and growth.

3 2. Expression of mevalonate pathway enzymes is controlled by the SREBP family of transcription factors.

4 3. In cancer cells, oncogenic signaling pathways deregulate the activity of the SREBP transcription

5 factors and mevalonate pathway enzymes.

6 4. Deregulated production of mevalonate pathway metabolites modulates multiple signaling pathways in

7 cancer cells and contributes to transformation.

8 5. Clinical trials evaluating the utility of mevalonate pathway inhibitors as anti-cancer agents have shown

9 responses in some, but not all, patients; discovering biomarkers to identify responders and developing

10 combination therapies will further enhance their utility.

11 6. Inhibiting the SREBP transcription factors is a promising strategy to increase the efficacy of

12 mevalonate pathway inhibitors as anticancer therapeutics, and also to potentially combat resistance. 
1 Cancer cells reprogram their metabolism to provide energy and essential building blocks required to

2 maintain their aberrant survival and growth ${ }^{1-5}$. This reprogramming may occur through mutations in

3 metabolic enzymes (e.g. isocitrate dehydrogenase ${ }^{6,7}$ ) or alterations in cell signaling due to oncogenic

4 events and/or the remodeled tumour microenvironment. These activated signaling cascades in turn

5 deregulate the expression ${ }^{8,9}$ and/or activity of enzymes in key metabolic pathways ${ }^{10}$, including the

6 mevalonate (MVA) pathway ${ }^{11}($ Fig.1A, 1B).

7 The MVA pathway uses acetyl-CoA, nicotinamide adenine dinucleotide phosphate (NADPH) and ATP to

8 produce sterols and isoprenoids that are essential for tumour growth ${ }^{12}$ (Fig.1A, 1B). Production of acetyl-

9 CoA occurs following glucose, glutamine or acetate consumption, which are often increased in cancer

10 cells $\mathrm{s}^{4,5,13,14}$. NADPH is produced from a variety of sources, including the pentose phosphate pathway,

11 malic enzyme and isocitrate dehydrogenases ${ }^{15,16}$. Therefore, the MVA pathway is highly integrated into

12 the overall metabolic state of cancer cells (Fig.1A). Transcription of MVA pathway genes is primarily

13 controlled by the sterol regulatory element-binding protein (SREBP) family of transcription factors.

14 When sterol levels are high, the SREBPs are maintained in an inactive state at the endoplasmic reticulum

15 (ER), where some MVA pathway enzymes are also localized. In response to sterol deprivation, a

16 feedback response is initiated that leads to the SREBPs, along with their binding partner SCAP (SREBP

17 cleavage activating protein), dissociating from the INSIGs (insulin induced genes) and translocating from

18 the ER to the Golgi (Fig.2). At the Golgi, the SREBPs are cleaved and translocate to the nucleus where

19 they bind to sterol regulatory elements (SREs) in the promoters of their target genes and activate the

20 transcription of MVA pathway genes to restore sterol and isoprenoid levels ${ }^{17}$.

21 The importance of MVA pathway metabolites to the survival of cancer cells is highlighted in recent

22 studies that have identified a large number of MVA pathway enzymes as essential for the survival of

23 several cancer cell lines ${ }^{18-20}$. Additionally, numerous studies have shown that the statin family of drugs,

24 which inhibit the initial flux-controlling enzyme of the MVA pathway, 3-hydroxy-3-methylglutaryl-CoA

25 reductase (HMGCR), decrease growth and increase apoptosis in many cancer types in vitro and in vivo ${ }^{21-}$ 
125 . These observations point to the MVA pathway being a key dependency in tumours, and one that is

2 readily targetable.

3 The MVA pathway has been suggested to be oncogenic in some studies. Early work in chronic

4 lymphocytic leukemia (CLL) showed that MVA can stimulate replication in primary leukemic cells ${ }^{26}$. In

5 an independent study, overexpressing the catalytic domain of HMGCR in primary mouse embryonic

6 fibroblasts cooperated with RAS to promote foci formation, suggesting that HMGCR is a metabolic

7 oncogene ${ }^{27}$. Also, the direct infusion of MVA into mice harbouring breast cancer cell xenografts caused

8 an increase in tumour growth ${ }^{28}$. Data from primary patient samples also suggest a role for the MVA

9 pathway in promoting tumorigenesis, with higher expression of MVA pathway genes correlating with

10 poor prognosis in breast cancer ${ }^{27}$. Collectively, this evidence indicates that the MVA pathway plays a key

11 role in cancer.

12 In this article, we review recent evidence demonstrating that the MVA pathway is deregulated in cancer

13 through aberrant cell signaling, which in turn establishes a tumour vulnerability that can be

14 therapeutically targeted to impact patient care and outcome.

\section{Mevalonate-derived metabolites in cancer}

16 Initially, the regulation and function of the MVA pathway and its metabolites was studied in the context

17 of normal and hypercholesterolaemic tissues, which led to the Nobel prize-winning discoveries of Bloch

18 and Lynen in $1964^{29}$, and later Brown and Goldstein in $1985^{11,30}$. In recent years, the importance of MVA

19 pathway-derived metabolites in cancer has become increasingly appreciated, and is discussed below.

20 Cholesterol. Cholesterol is an important component of most cellular membranes. Highly proliferative

21 cancer cells need to rapidly produce membranes, and an increase in cholesterol synthesis contributes to

22 this process. Cholesterol is also an integral component of lipid rafts, which are necessary to form

23 signaling complexes ${ }^{31-33}$. The cholesterol content of the ER has recently been linked to the antiviral type I

24 interferon (IFN) response, with low ER cholesterol triggering an IFN response in macrophages that 
1 protects mice from viral challenge ${ }^{34}$. It is therefore possible that high cholesterol, produced by the MVA

2 pathway, could play a role in protecting cancer cells from immune surveillance and immunotherapies ${ }^{35,36}$.

3 Cholesterol also serves as the precursor for downstream products, such as steroid hormones and

4 oxysterols: steroid hormones drive the initiation and progression of cancers such as breast and prostate

5 carcinomas $^{37}$; increased oxysterol production can activate the liver X receptors (LXRs), which have been

6 proposed to be a therapeutic target in multiple cancer types ${ }^{38,39}$.

7 Cancer cells therefore require cholesterol for growth and survival, and lowering intracellular cholesterol

8 biosynthesis is a promising anti-cancer strategy.

9 Isopentenyl-diphosphate. In human cells, the MVA pathway is the sole intracellular source for

10 isopentenyl-diphosphate (IPP) $(\text { Fig. 1B })^{40}$. Aberrant activation of the MVA pathway in cancer results in

11 elevated intracellular levels of IPP, which has been shown to activate host $\gamma \delta$ T cells that subsequently kill

12 the IPP-overexpressing cells ${ }^{41,42}$. These observations led to phase I clinical trials that evaluated the in vivo

13 expansion of $\gamma \delta \mathrm{T}$ cells in response to zoledronate, a bisphosphonate that inhibits the MVA pathway

14 downstream of IPP (Table 1), in combination with IL-2 treatment in advanced-stage breast ${ }^{43}$ and

15 prostate ${ }^{44}$ cancer. In both studies, the therapy was well-tolerated and the number of sustained peripheral

$16 \gamma \delta$ T cells was correlated with improved clinical outcome $e^{41,43,44}$. Future phase II clinical trials will reveal

17 whether combined zoledronate and IL-2 therapy is an effective anti-cancer strategy.

18 Farnesyl-and geranylgeranyl-diphosphate. Farnesyl-diphosphate (FPP) and geranylgeranyl-diphosphate

19 (GGPP) are produced by sequential condensation reactions of dimethylallyl-diphosphate with two or three

20 units of IPP, respectively. FPP and GGPP are hydrophobic chains that are essential for the isoprenylation

21 of proteins. This post-translational modification (PTM) tethers proteins to cell membranes, enabling

22 proper protein localization and function ${ }^{45-48}$. Most small GTPases, like RAS and RHO, are

23 isoprenylated ${ }^{49}$, and many are involved in tumourigenesis. Inhibiting the MVA pathway can reduce the

24 isoprenylation of RAS, RHO and other small GTPases ${ }^{50-52}$, and leads to cell death in some cancer cells. 
1 This cell death can be reversed by the addition of GGPP, and sometimes FPP, suggesting that these MVA

2 pathway metabolites are essential for tumour cell viability ${ }^{52-56}$. Evidence suggests it is unlikely that any

3 one isoprenylated protein can be assigned functional responsibility for this cancer cell dependency on

4 GGPP and FPP ${ }^{52,57}$; instead, it appears that this is a 'class effect', with depletion of these isoprenoid pools

5 potentially affecting the many proteins that are isoprenylated ${ }^{58}$. Despite this dependency, directly

6 inhibiting the isoprenylation of proteins by geranylgeranyl transferase inhibitors (GGTIs) or farnesyl

7 transferase inhibitors (FTIs) has not been a successful anti-cancer strategy to date ${ }^{59}$. The rationale behind

8 these drug development programs was that key isoprenylated onco-proteins, like RAS, could be targeted.

9 However, the efficacy of FTIs was impeded by alternate isoprenylation using GGPP, and GGTIs have

10 been disappointingly toxic ${ }^{60,61}$. Further development of next generation FTIs and GGTIs remains a

11 relatively limited and focused area of research ${ }^{59,62-66}$.

12 Dolichol. Dolichol is derived from an 18-20mer of IPP, and is an essential component for the $N$ -

13 glycosylation of nascent polypeptides in the $\mathrm{ER}^{67,68}$. Protein $\mathrm{N}$-glycosylation is frequently altered in

14 cancer and can contribute to tumour formation, proliferation and metastasis ${ }^{69}$. Not all $N$-glycans are

15 associated with tumour progression; the complex branching of $\mathrm{N}$-glycans leads to tumour suppressive

16 properties in some cancers (reviewed $\mathrm{in}^{69}$ ). Glucose-derived $N$-acetylglucosamine has recently been

17 shown to be necessary for the $N$-glycosylation of SCAP prior to ER-to-Golgi translocation. The

18 SCAP/SREBP complex therefore remains inactive in the ER when glucose is absent, even in the presence

19 of low sterols ${ }^{70}$.

20 Coenzyme $Q$. Together with quinone groups, isoprenoids are also used to produce coenzyme $\mathrm{Q}(\mathrm{CoQ})$.

21 The hydrophobic isoprenoid chain localizes CoQ to the inner membrane of the mitochondria, where the

22 quinone group acts to transfer electrons from complex I or II to complex III of the electron transport

23 chain, thus enabling ATP production ${ }^{71}$. CoQ is therefore critical for ATP production in those cancer cells

24 that rely on oxidative phosphorylation to produce energy ${ }^{72,73}$. 
2 Intracellular pools of MVA pathway metabolites are tightly regulated by modulating the expression and

3 activity of the MVA pathway enzymes. MVA pathway gene expression is mainly controlled by the

4 SREBP transcription factors (Fig.2). There are three SREBP proteins, transcribed from two genes:

5 SREBP2 is transcribed from the $S R E B F 2$ gene, and is the main transcription factor for MVA pathway-

6 associated genes; SREBP1a and SREBP1c are transcribed from alternate start sites in the SREBF1 gene,

7 with SREBP1a regulating the expression of both MVA and fatty acid metabolism genes, and SREBP1c

8 predominantly regulating the expression of fatty acid synthesis genes ${ }^{74,75}$. ChIP-seq studies have

9 indicated some overlap in the target genes of each SREBP, including MVA pathway genes, affording

10 some redundancy ${ }^{76,77}$. Most work also shows an overlap in the regulation of the SREBPs; however, the

11 majority of studies limit full characterization to SREBP1, and most do not distinguish between SREBP1a

12 and SREBP1c due to antibody specificity. Given the importance of the MVA pathway in cancer, a

13 complete characterization of SREBP2 in transformed cells is needed.

14 In recent years, oncogenic and tumour-suppressive pathways have been shown to converge on the MVA

15 pathway and its regulatory feedback loop. Cancer cells, with their aberrant growth and metabolism, are

16 therefore primed to upregulate the MVA pathway to provide essential building blocks for continued

17 proliferation. The integration of cellular signaling from growth factors and essential metabolites, with the

18 regulation of the MVA pathway and its SREBP-regulated feedback response, highlights the importance of

19 this pathway in cancer cells.

20 PI3K/AKT. The PI3K/AKT signaling pathway is a major regulator of cell survival and proliferation in

21 response to growth factors. It is the single most frequently altered pathway in cancer, and PIK3CA is the

22 second most frequently mutated gene ${ }^{78}$. Inactivating mutations in its negative regulator PTEN, and/or

23 hyperactivity of receptor tyrosine kinases are also frequent in cancer. Alterations in this pathway

24 generally act to augment PI3K/AKT signaling, and consequently increase proliferation of cancer cells. 
1 PI3K/AKT can activate the MVA pathway by a variety of mechanisms (Fig.3). For example, stimulation

2 of PI3K/AKT signaling by growth factors, such as insulin, PDGF or VEGF, can increase the mRNA and

3 protein expression of SREBP1 and SREBP2 ${ }^{79-83}$. It should be noted that while PI3K/AKT signaling

4 strongly and consistently increases the mRNA and protein levels of SREBP1a and 1c, its effects on

5 SREBP2 expression are context-dependent. AKT, alternatively known as PKB, has also been suggested

6 to increase the stability of nuclear SREBP1a, SREBP1c and SREBP2 by preventing their FBXW7-

7 mediated degradation ${ }^{84}$. FBXW7 is an E3 ubiquitin ligase that binds to and ubiquitylates phosphorylated

8 SREBPs, leading to their proteasomal degradation. The importance of this degradation pathway is

9 highlighted by an increase in cholesterol and fatty acid synthesis in FBXW7-deficient cells ${ }^{84}$. The

10 residues that are recognized by FBXW7 are phosphorylated by GSK-3 $\beta$, and AKT has been suggested to

11 inhibit this phosphorylation and prevent FBXW7-mediated degradation of the SREBPs (Fig.3). Insulin

12 also causes the dissociation of INSIG from SCAP/SREBP1c in a sterol-independent manner, leading to

13 increased transcription of MVA pathway genes ${ }^{85-88}$. These studies were further validated through genetic

14 approaches, where SREBP1 and SREBP2 expression and activity were increased with expression of

15 constitutively active PI3K or AKT, and abrogated by dominant-negative AKT ${ }^{80,88,89}$. The increase in lipid

16 and cholesterol production mediated by the PI3K/AKT/SREBP axis promotes proliferation of cancer cells

17 and tumorigenesis in vitro and in vivo ${ }^{90-92}$. Conversely, inhibiting the MVA pathway decreases PI3K

18 activity $^{93}$, possibly through decreased RAS isoprenylation ${ }^{93,94}$, demonstrating a two-way regulatory

19 relationship between PI3K/AKT signaling and the MVA pathway.

20 Increased MVA pathway activity is inconsequential without the availability of both acetyl-CoA and

$21 \mathrm{NADPH}$, and PI3K/AKT signaling meets this requirement by increasing glucose uptake and the rate of

22 glycolysis in cancer cells ${ }^{95}$. This is important as acetyl-CoA is also used by other processes, such as fatty

23 acid synthesis and protein acetylation ${ }^{13}$. Thus, PI3K/AKT signaling couples substrate availability with the

24 activity of the MVA pathway in cancer. 
mTORC1. Downstream of PI3K/AKT signaling, mTOR complex 1 (mTORC1) acts as a sensor of growth

2 signals (such as insulin) and nutrients (such as amino acids) to regulate cellular growth ${ }^{96}$. It is often

3 deregulated in cancer, and this supports aberrant growth. mTORC1 increases mRNA translation by

4 phosphorylating and activating ribosomal S6 kinase $1(\mathrm{~S} 6 \mathrm{~K} 1)^{97,98}$ and repressing the activity of the

5 inhibitor of cap-dependent translation, eIF4E-binding protein 1 (4E-BP1) ${ }^{99}$. SREBPs are major

6 downstream effectors of mTORC1 signaling, as evidenced by increased lipogenesis in response to

7 mTORC1 activation ${ }^{100-102}$. The observation that SREs are the most common regulatory elements in

8 mTORC1-induced genes further strengthens the link between mTORC1 and the SREBPs ${ }^{102}$. This link is

9 also evident in primary breast cancer patient samples, where patients with high levels of phosphorylated

10 S6K1 had corresponding high expression of SREBP target genes such as FASN, LDLR and MVK ${ }^{90}$. This

11 study also compared protein from tumour and adjacent normal breast samples, and described an increase

12 in FASN protein levels in the tumours that had higher levels of phosphorylated S6K1.

mTORC1 can regulate the SREBP transcription factors at multiple levels, although there are some cell-

14 and tissue-type differences (Fig.3). S6K1 has been shown to activate SREBP2 processing and increase

15 expression of MVA pathway genes in a hepatocellular carcinoma cell line, although the mechanism

16 remains unclear ${ }^{103}$. Greater understanding of the role of mTORC1 in SREBP activity came with the

17 development of torins, which are catalytic site mTOR inhibitors ${ }^{104}$. The original allosteric mTOR inhibitor, rapamycin, prevents phosphorylation of S6K1 but does not inhibit 4E-BP1 phosphorylation equally in all systems. In contrast, catalytic site inhibitors, like torins, inhibit the phosphorylation of

20 multiple mTOR targets, including S6K1 and 4E-BP1 ${ }^{104,105}$. Recent work comparing torin and rapamycin

21 action implicated a role for LIPIN1 in mediating the effects of mTORC1 on the SREBPs ${ }^{106}$. LIPIN1 is a

22 nuclear phosphatidic acid phosphatase that is inhibited by direct phosphorylation by mTORC1,

23 independent of S6K1. Active, unphosphorylated LIPIN1 indirectly prevents the transcription of SREBP

24 target genes, although the mechanism remains unclear. A further link between LIPIN1 and the MVA

25 pathway was uncovered in studies using skeletal muscle, in which statins and LIPIN1 were shown to 
1 increase autophagy ${ }^{107}$. Given the role of SREBP2 in transcribing numerous autophagy genes ${ }^{77,}{ }^{108}$, further

2 work is needed to fully understand the interplay between mTORC1, LIPIN1 and the SREBPs.

3 The position of the SREBPs as key effectors of mTORC1 signaling presents a potential vulnerability in

4 tumours that have deregulated mTORC1 activity. Previous studies have linked the loss of SREBPs in

5 breast cancer to the induction of ER stress, which induced apoptosis through TTOR $^{109}$. A separate study

6 showed that genetic knockdown of SREBPs reduced proliferation and increased cell death in mTORC1-

7 activated breast cancer cell lines ${ }^{90}$. The observation that double knockdown of SREBP1 and SREBP2

8 showed the greatest pro-apoptotic effect suggests that small molecule inhibitors that target both SREBP1

9 and SREBP2 will have the greatest therapeutic benefit.

10 AMPK. Playing an opposing role to mTORC1, AMP-activated protein kinase (AMPK) acts to dampen

11 anabolic pathways when intracellular ATP levels are low. This role as an energy sensor and central

12 regulator of metabolism is critical in metabolic disorders such as type II diabetes and cancer ${ }^{110}$. AMPK

13 was discovered through its ability to phosphorylate and reduce the activity of microsomal HMGCR in rat

14 liver extracts ${ }^{111,112}$. Further studies showed AMPK phosphorylates S872 within the catalytic domain of

15 HMGCR, inhibiting its enzymatic activity in a manner that is independent of its feedback regulation by

16 MVA pathway metabolites ${ }^{113,114}$. The SREBPs are also direct targets of AMPK phosphorylation ${ }^{115}$.

17 Activated AMPK specifically interacts with both the precursor and nuclear forms of the SREBP1c and

18 SREBP2, and phosphorylation by AMPK inhibits SREBP proteolytic processing and transactivation

19 activity $^{115}$. Activation of AMPK in HepG2 cells by either polyphenols or metformin has been shown to

20 stimulate this phosphorylation, which suppressed the accumulation of SREBPs in the nucleus under

21 hyperglycemic and hyperinsulinemic conditions ${ }^{115}$. Moreover, activation of AMPK in the livers of

22 insulin-resistant mice inhibited the transcription of enzymes involved in lipid and cholesterol

23 biosynthesis, including the MVA pathway enzymes HMGCS1 and HMGCR, which consequently resulted

24 in a decrease in hepatic triglyceride and cholesterol levels ${ }^{115}$. AMPK can therefore inhibit MVA pathway

25 activity directly via phosphorylation of HMGCR, and indirectly through the phosphorylation and 
1 repression of the SREBPs. However, the relevance of this regulation in the context of cancer is poorly

2 understood.

3 The MVA pathway may also play a role in regulating AMPK activity, thereby forming a regulatory

4 feedback loop. The tumour suppressor liver kinase B1 (LKB1), which phosphorylates and activates

5 AMPK, is farnesylated at a highly conserved C-terminal CAAX motif ${ }^{116,117}$. Knock-in mice expressing a

6 mutant LKB1, which could not be farnesylated, exhibited reduced membrane-bound LKB1 and impaired

7 AMPK activity ${ }^{117}$. This hints at a negative feedback loop, whereby activation of AMPK in response to

8 decreased cellular energy results in the inhibition of the MVA pathway via the phosphorylation of

9 HMGCR and the SREBPs. This in turn reduces the FPP pool within the cell, thereby hindering LKB1

10 farnesylation and inhibiting AMPK activation.

11 p53 and pRB. The p53 tumour suppressor is one of the most frequently altered genes in cancer, and

12 mutations within the coding region of this gene can confer oncogenic properties to the p53 protein

13 product. Two gain-of-function mutations ( $\mathrm{p} 53^{\mathrm{R} 273 \mathrm{H}}$ and $\mathrm{p} 53^{\mathrm{R} 280 \mathrm{~K}}$ ) enable $\mathrm{p} 53$ to functionally interact with

14 nuclear SREBP2 and increase transcription of MVA pathway genes (Fig.4). This MVA pathway gene

15 activation was necessary and sufficient for mutant p53 to disrupt normal breast acinar morphology ${ }^{118}$, and

16 mutant p53 expression in primary breast cancer tissues was correlated with elevated expression of sterol

17 biosynthesis genes. Conversely, wild type p53 can reduce lipid synthesis under conditions of glucose

18 starvation ${ }^{119}$ by inducing the expression of LIPIN1, which, as described above, can prevent the

19 association of SREBPs with chromatin ${ }^{106}$. The interplay between p53 and the MVA pathway suggests that

20 the MVA pathway may be a novel therapeutic target for tumours, particularly breast cancers that harbour

21 p53 gain-of-function mutations.

22 The tumour suppressor protein retinoblastoma $(\mathrm{pRB})$ has also been implicated as a regulator of the MVA

23 pathway (Fig.4). In a mouse model of C-cell adenoma, $R b$ loss resulted in enhanced isoprenylation and

24 activation of N-RAS ${ }^{120}$. Loss of pRB relieved suppression of the transcription factors E2F-1 and E2F-3, 
1 which were shown to bind and activate the promoters of numerous prenyltransferase genes, farnesyl

2 diphosphate synthase $(F d p s)$ and $\operatorname{Srebf} 1^{120}$. Moreover, pRB prevented the association of SREBP1 and

3 SREBP2 with the Fdps gene promoter ${ }^{120}$, suggesting that pRB negatively regulates the MVA pathway at

4 both the transcriptional and post-translational level.

5 MYC. The MYC transcription factor is a potent oncogene that can drive transformation in multiple cancer

6 types. It is deregulated in over $50 \%$ of cancers, and can reprogram cancer cell metabolism to enable

7 proliferation and survival of cancer cells ${ }^{121-124}$. Like the SREBPs, it is a bHLH-LZ protein, and has been

8 shown to bind to SREBP1 to drive somatic cell reprogramming into induced pluripotent stem cells ${ }^{125}$.

9 Analysis of data from the ENCODE project ${ }^{126}$ also shows that MYC binds to promoters of MVA pathway

10 genes, in close proximity to SREBP1 and SREBP2 binding regions, suggesting that MYC can contribute

11 to the expression of MVA pathway enzymes (Fig.4). As the MVA pathway is essential for cancer cells,

12 and MYC has a major role in metabolic regulation, MYC may ensure that MVA pathway metabolites are

13 not limiting for tumorigenesis. The MVA pathway was also shown to be important in a MYC-driven

14 transgenic model of hepatocellular carcinoma. In that study, atorvastatin reduced tumour initiation and

15 growth, possibly through reduced isoprenylation of RAC1 leading to activation of PP2A, a negative

16 regulator of $\mathrm{MYC}^{127}$. More recently, $M y c$ haploinsufficient mice were shown to have an increased

17 lifespan, which was associated with decreased expression of MVA pathway genes, including Hmgcr and

18 Srebf $2^{128}$. Given the importance of MYC in driving cancer, and the difficulty in targeting it

19 therapeutically, further work is warranted to uncover the relationship between MYC and the MVA

20 pathway.

\section{Signaling from the MVA pathway}

22 Altered metabolism in tumours not only fulfills the energetic and biosynthetic needs of a dividing cell, but

23 also produces metabolites important for downstream signaling. This is particularly true of the isoprenoid 
1 and sterol metabolites produced by the MVA pathway, which are also used by cancer cells to modulate

2 multiple downstream signaling pathways that are important for tumour progression.

$3 \quad$ YAP/TAZ. It was recently shown that the oncogenes YAP and TAZ require the MVA pathway to be fully

4 functional ${ }^{129}$. YAP and TAZ are transcriptional co-activators that facilitate the transcriptional activation

5 of pro-growth genes and repression of pro-apoptotic genes. The nuclear localization of YAP/TAZ is

6 negatively regulated, in part, by activation of the tumour-suppressive Hippo signaling pathway.

7 Activation of the Hippo cascade results in the phosphorylation and activation of the LATS1/2 kinases,

8 which phosphorylate YAP and TAZ and retain them in the cytoplasm. YAP and TAZ nuclear localization

9 requires the MVA pathway ${ }^{129}$ (Fig.5). Concurrent knockdown of SREBF1 and SREBF2 reduced nuclear

10 localization of YAP and TAZ ${ }^{129}$. These effects were mimicked by GGTIs, and prevented by a RHOA

11 mutant that does not require geranylgeranylation ${ }^{129}$. This suggests that SREBP-mediated induction of the

12 MVA pathway maintains intracellular GGPP pools, which is necessary for RHOA activity and YAP/TAZ

13 nuclear localization. However, it is unclear whether these effects are dependent on Hippo signaling.

14 While some studies showed that MVA pathway-mediated YAP/TAZ signaling is independent of

15 LATS1/2 via RNAi-knockdown experiments ${ }^{129,130}$, one study demonstrated that atorvastatin or GGTI

16 treatment increases phosphorylation of LATS1/2, suggesting that geranylgeranylation regulates Hippo

17 signaling ${ }^{131}$. A separate study reported constitutive SREBP activation in the livers of mice with a liverspecific LATS2 deletion, which corresponded to an increase in liver free cholesterol and protection from

19 p53-mediated apoptosis ${ }^{132}$.

20 Activation of the MVA pathway and YAP/TAZ are correlated with mutant p53 expression in primary

21 tumours, suggesting a dysfunctional mutant p53/SREBP/YAP/TAZ axis in cancer ${ }^{129}$. Overexpression of

$22 \mathrm{p} 53^{\mathrm{R} 280 \mathrm{~K}}$ in a p53-null cell line activated YAP/TAZ only when the MVA pathway was active, placing the

23 MVA pathway as a critical intermediate in the oncogenic activation of YAP/TAZ by mutant p53 ${ }^{129}$. 
1 Hedgehog. Cholesterol plays a multifaceted role in regulating cell signaling. For example, the Hedgehog

2 (Hh) signaling pathway, which plays important roles in vertebrate development and tumorigenesis, is

3 regulated by sterols at multiple levels ${ }^{133}$. Cholesterol itself can serve as a substrate for the post-

4 translational modification of Hh ligands, which is required for their proper trafficking ${ }^{134}$. Cholesterol and

5 cholesterol-derived oxysterols can also activate $\mathrm{Hh}$ signal transduction in medulloblastoma, whereas

6 inhibiting the MVA pathway or downstream sterol biosynthesis decreased Hh signaling and reduced cell

7 proliferation $^{135}$ (Fig.5).

8 Steroid hormone signaling. Cholesterol also serves as the precursor for steroid hormones, which drive

9 the initiation and progression of cancers such as hormone-dependent breast and prostate cancer. In breast

10 cancer, patients with oestrogen receptor alpha (ER $\alpha)$-positive disease are commonly treated with

11 aromatase inhibitors. Recent work demonstrated that long-term oestrogen deprivation of ER $\alpha$-positive

12 breast cancers led to stable epigenetic activation of the MVA pathway and cholesterol biosynthesis,

13 coupled with increased SREBP occupancy on open chromatin ${ }^{136}$. The resulting elevated levels of $27-$

14 hydroxycholesterol was sufficient to activate ER $\alpha$ signaling in the absence of exogenous oestrogen,

15 driving the activation of genes that promote an invasive cell phenotype ${ }^{136}$. Similarly, in prostate cancer,

16 the de novo synthesis of androgens from cholesterol drives androgen receptor (AR) activity in castration-

17 resistant disease ${ }^{137}$ (Fig.5). This, coupled with the observations that SREBP expression is elevated in

18 advanced-stage prostate cancer ${ }^{138,139}$, suggests a role for the MVA pathway in prostate cancer

19 progression. These findings warrant further investigation into the utility of inhibitors of the MVA

20 pathway and/or SREBPs for the treatment of hormone-driven cancers.

\section{Targeting the MVA pathway in cancer.}

22 As outlined above, multiple oncogenic signaling pathways can deregulate the MVA pathway for

23 enhanced cell survival and growth. In turn, MVA pathway activity is required to regulate the downstream

24 propagation of many cell signals. These, coupled with the essentiality of several MVA pathway genes in 
cancer cells, suggest that the MVA pathway is a tumour vulnerability that can be targeted as part of a

2 therapeutic strategy to treat cancer. The most promising way to block this pathway in tumours is to inhibit

3 HMGCR using statins, although inhibiting other flux-control points may also have anti-cancer benefits ${ }^{17}$.

4 Statins have been safely used for decades to treat patients with hypercholesterolaemia ${ }^{140}$, and although

5 epidemiological evidence has been mixed, the majority of reports indicate that statin use is correlated with

6 reduced mortality in multiple cancer types ${ }^{141-143}$. Evidence also suggests that certain stages of cancer

7 progression, such as breast cancer recurrence, are particularly sensitive to the anti-cancer activities of

8 statins $^{141,144-146}$. Although the cholesterol-lowering effects of statins are due to inhibition of MVA

9 pathway activity in the liver, lipophilic statins such as atorvastatin, simvastatin and lovastatin have been

10 detected in extra-hepatic tissues such as the brain, in both the active acid and inactive lactone forms ${ }^{147}$. In

11 contrast, the hydrophilic pravastatin could only be detected in the liver ${ }^{147}$, suggesting that hydrophilic

12 statins may be clinically limited as anticancer agents. It is currently unknown whether lipophilic statins

13 accumulate in tumour tissues at concentrations that are cytotoxic to cancer cells (reviewed in ${ }^{148}$ ). Efforts

14 are underway to directly address this issue, and to determine the clinical utility and recommended dose of

15 statins when used as anti-cancer therapeutics.

16 Many studies have shown that statins can directly and specifically trigger apoptosis of tumour cells $\mathrm{s}^{53,149-}$

$17 \quad{ }^{152}$. For example, statins trigger apoptosis of cells derived from acute myelogenous leukemia (AML),

18 while normal myeloid progenitors do not undergo apoptosis and retain full proliferative potential ${ }^{25}$. This

19 tumour-normal index may be due to the altered metabolic reprogramming of tumour cells leading to an

20 increased dependence on MVA pathway metabolites for growth and survival. The widespread use of

21 statins for cholesterol management also demonstrates that these drugs cause minimal damage to normal

22 cells. Side-effects are regularly treated by switching to a different statin or potentially by co-treating with

$23 \mathrm{CoQ}$, although the latter is controversial due to conflicting clinical evidence ${ }^{153,154}$.

24 This suggests that statins possess a high therapeutic index to target tumours in vivo, despite the ubiquitous

25 expression of the MVA pathway. This rationale has led to multiple clinical trials investigating the efficacy 
1 of various statins as a therapeutic option in a variety of tumour types. Two recent breast cancer window-

2 of-opportunity clinical trials, using atorvastatin ${ }^{155}$ or fluvastatin ${ }^{156}$, showed reductions in the Ki67 index in

3 a subset of patients administered cholesterol-management doses of statins between diagnosis and surgery.

4 Statins have also been safely used in combination with other agents to increase efficacy. For example,

5 pravastatin was combined with standard-of-care in hepatocellular carcinoma and AML, resulting in

6 significantly longer median survival ${ }^{157}$ and complete or partial response in $60 \%$ of patients ${ }^{158}$,

7 respectively. In another study, combining lovastatin with thalidomide and dexamethasone in patients with

8 relapsed or refractory multiple myeloma (MM) led to prolonged overall survival and progression-free

$9 \quad$ survival $^{159}$.

10 Despite evidence of patient response to statins as anti-cancer agents, many other patients remained non-

11 responsive to statin treatment in other cancer clinical trials ${ }^{160}$. This is consistent with the current paradigm

12 of tumour heterogeneity. This lack of response might also be expected considering the evidence we have

13 laid out above showing that the MVA pathway is regulated by many key oncogenic signals. Like many

14 anti-cancer agents, a personalized medicine approach is needed to implement statins, and/or other

15 inhibitors of the MVA pathway, as a successful class of therapeutics. To this end, a molecular signature of

16 basal mRNA expression has been developed for breast cancer $^{22}$ and deregulated MYC expression has

17 been a proposed indicator of statin response in specific tumour-types ${ }^{161}$; however, essential follow-

18 through validation is required. At this time, it is difficult to predict which cancers will be particularly

19 sensitive to statin therapy. In addition to AML and MM (Table 1), encouraging results from both clinical

20 trials ${ }^{155,156}$ and epidemiological ${ }^{162,163}$ studies suggest patients with hormone-dependent cancers, such as

21 breast and prostate, may benefit from the addition of statins to their treatment regimen. This may be in

22 part because the MVA pathway end-product cholesterol is the precursor for hormones such as oestrogen

23 and androgens, which play a major role in the development of these types of cancers. Hepatocellular

24 carcinoma also appears particularly responsive to statins ${ }^{157}$, perhaps because of the hepatotropic 
1 pharmacology of this family of drugs. Clinical trials are required in these and other cancers to further

2 define the subset of cancers that are particularly statin-sensitive.

3 Critical to the regulation of the MVA pathway is the tightly-controlled, SREBP-mediated feedback

4 mechanism, where inhibition of the MVA pathway results in the activation of the SREBPs and an

5 increase in the expression of MVA pathway genes, an effect that may be amplified in cancer cells.

6 SREBP activation also increases the expression of the low-density lipoprotein receptor (LDLR), which

7 leads to increased uptake of exogenous, lipoprotein-derived, cholesterol; an effect that has been shown to

8 be important in cancer cells ${ }^{164-167}$. The SREBPs therefore function to replenish MVA pathway

9 metabolites, which can dampen the apoptotic response following statin treatment. This would be a classic

10 resistance mechanism, similar to what is seen with other anti-cancer therapeutics such as BRAF inhibitors

11 in BRAF-mutant melanoma. Cells treated with BRAF inhibitors, such as vemurafenib, can acquire an

12 activating mutation in downstream kinases (e.g. MAP2K1) or increase in expression of receptor tyrosine

13 kinases (e.g. EGFR), bypassing the need for BRAF activity ${ }^{168}$. These studies demonstrate that inhibiting

14 both the cancer vulnerability and the resistance/feedback mechanism is crucial for maximum efficacy ${ }^{169}$.

15 Hence, inhibiting the SREBP-regulated feedback response in conjunction with statin therapy could

16 prevent resistance, thereby increasing the efficacy of statins as anti-cancer agents and the number of

17 responsive patients (Fig.6).

18 Evidence that targeting the SREBPs in combination with statin therapy is a viable strategy has been

19 provided by several recent studies. Firstly, a study looking at breast and lung cancer cell lines performed

20 an shRNA screen to uncover genes that, when knocked down, potentiated the pro-apoptotic effects of

21 statins $^{170}$. The MVA pathway genes HMGCS1, GGPS1, SCAP and SREBF2 all scored highly, adding

22 credence to either inhibiting other enzymes in the MVA pathway or inhibiting the SREBP-mediated

23 feedback response in combination with statin therapy. A second study showed that statin-induced SREBP

24 processing can be blocked by another approved agent, dipyridamole ${ }^{51}$. Mechanistically, dipyridamole

25 reduced the transcription of SREBP target genes such as HMGCS1 and HMGCR, and synergized with 
1 statins to increase apoptosis in AML and MM cell lines and patient samples. Other compounds, such as

2 tocotrienols, have also been demonstrated to synergize with statins to induce cancer cell apoptosis ${ }^{171}$, an

3 effect that may be associated with their ability to degrade nuclear SREBP2 and inhibit its transcriptional

4 activity $^{172}$. Although a number of other small molecules, including fatostatin, have been shown to inhibit

5 SREBP processing, their lack of approval for use in patients limits their potential to immediately impact

6 cancer patient care $\mathrm{e}^{173-175}$. Therefore, at this time, clinical investigation into the utility of combined statins

7 and SREBP inhibitors for the treatment of cancer is warranted (Table 1).

\section{Outlook.}

9 Understanding tumour metabolism in the context of oncogenic signals has the potential to drive the development of targeted personalized therapies. The various signaling pathways that we have described in

11 this review are important drivers in a majority of cancers, and they all have the ability to deregulate the

12 MVA pathway, making those cancers potentially vulnerable to MVA pathway inhibition. Whether this

13 occurs in every patient that presents with these lesions remains unclear. More work is needed to

14 understand the extent to which driver mutations increase flux through the MVA pathway in patients.

15 Rapidly developing technologies for the comprehensive flux-based analysis of MVA pathway metabolites

16 will provide further advances in understanding how the MVA pathway receives and responds to

17 oncogenic signals. In patients, it may be more feasible to determine pathway activity by mapping their

18 oncogenic lesions to their sterol feedback response at the protein level (via SREBP localization) or

19 mRNA expression level, which may identify patients who will respond to MVA pathway inhibition.

20 Designing clinical trials that will identify potential responders prior to treatment is needed to prevent

21 expensive failures of therapies that may still have benefits to a subset of patients. Improving reagents,

22 particularly antibodies to HMGCR and SREBP2, will also aid trial design and interpretation. 
1 The essentiality of the MVA pathway in many cancers, coupled with affordable and safe drugs that can

2 target it and its feedback response, provides a strong rationale to continue exploring this key metabolic

3 pathway in cancer.

4

5

6

7 Glossary.

8 Acetyl-CoA.

9 An essential metabolite that is used to drive many cellular processes, including the TCA cycle, fatty acid

10 and sterol biosynthesis, and acetylation of histones.

11 INSIG.

12 INSIG1 and INSIG2 interact with SCAP under sterol-rich conditions. They prevent SREBP activation by

13 retaining the SCAP/SREBP complex in the ER. They also promote the sterol-regulated degradation of

14 HMGCR.

15 SCAP.

16 Essential for SREBP ER-to-Golgi translocation. SCAP contains a sterol-sensing domain, and undergoes a

17 conformational change when sterols are low. This change causes a dissociation of the SREBP/SCAP

18 complex from INSIG.

$19 S 1 P / S 2 P$. 
1 Two proteases that cleave the SREBPs, and other proteins such as ATF6, in the Golgi. S1P cleaves at the

2 luminal loop of the SREBPs, whereas S2P is a hydrophobic protein that cleaves the SREBPs at a

3 transmembrane residue.

$4 \quad$ Sterol response element (SRE).

5 Motifs found in the promoters of genes that are transcribed in response to sterol deprivation. SREs are

6 necessary for the transcription of MVA pathway genes by the SREBPs.

\section{Isoprenylation.}

8 The attachment of a hydrophobic farnesol or geranygeraniol to the $\mathrm{C}$-terminus of proteins that contain a

9 CAAX motif, which anchors the proteins to lipid membranes. Geranylgeraniol can also be attached to

10 non-CAAX motif-containing proteins.

\section{Dipyridamole.}

12 A clinically-approved drug used to prevent platelet aggregation. A recent study showed that it also

13 prevents cleavage of SREBP2, potentiating the anti-cancer effects of statins, although the mechanism is

14 not yet known.

\section{Acknowledgements.}

16 We thank Jenna van Leeuwen and William Tu for helping to prepare this review. We also thank other

17 current and former members of the Penn lab for their helpful comments, including Alex Pandyra, Emily

18 Chamberlain, Jason DeMelo, Dharmesh Dingar, Ashley Hickman, Manpreet Kalkat, Corey Lorenco,

19 Diana Resetca and Aaliya Tamachi. We also acknowledge the many important contributions by our

20 colleagues that could not be cited here owing to space and reference constraints. The funding agencies

21 that enable our research include the Ontario Institute for Cancer Research through funding provided by

22 the Province of Ontario, the Canadian Institute for Health Research, the Department of Defense Breast 
1 Cancer Research Program, the Princess Margaret Cancer Foundation Hold'em for Life Prostate Cancer

2 Research Fund, and the Terry Fox Foundation Canada. 
1. Boroughs, L.K. \& DeBerardinis, R.J. Metabolic pathways promoting cancer cell survival and growth. Nat Cell Biol 17, 351-9 (2015).

2. Son, J. et al. Glutamine supports pancreatic cancer growth through a KRAS-regulated metabolic pathway. Nature 496, 101-5 (2013).

3. Possemato, R. et al. Functional genomics reveal that the serine synthesis pathway is essential in breast cancer. Nature 476, 346-50 (2011).

4. Comerford, S.A. et al. Acetate dependence of tumors. Cell 159, 1591-602 (2014).

5. Mashimo, T. et al. Acetate is a bioenergetic substrate for human glioblastoma and brain metastases. Cell 159, 1603-14 (2014).

6. Mardis, E.R. et al. Recurring mutations found by sequencing an acute myeloid leukemia genome. N Engl J Med 361, 1058-66 (2009).

7. Parsons, D.W. et al. An integrated genomic analysis of human glioblastoma multiforme. Science 321, 1807-12 (2008).

8. Christofk, H.R. et al. The M2 splice isoform of pyruvate kinase is important for cancer metabolism and tumour growth. Nature 452, 230-3 (2008).

9. Patra, K.C. et al. Hexokinase 2 is required for tumor initiation and maintenance and its systemic deletion is therapeutic in mouse models of cancer. Cancer Cell 24, 213-28 (2013).

10. Adam, J., Yang, M., Soga, T. \& Pollard, P.J. Rare insights into cancer biology. Oncogene 33, 254756 (2014).

11. Goldstein, J.L. \& Brown, M.S. Familial hypercholesterolemia: identification of a defect in the regulation of 3-hydroxy-3-methylglutaryl coenzyme A reductase activity associated with overproduction of cholesterol. Proc Natl Acad Sci U S A 70, 2804-8 (1973).

This manuscript is the first to suggest that a genetic abnormality could lead to the dysregulation of HMGCR and result in a defect in the regulation of cholesterol synthesis and contributed to Goldstein and Brown winning the Nobel Prize in Physiology or Medicine in 1985.

12. Clendening, J.W. \& Penn, L.Z. Targeting tumor cell metabolism with statins. Oncogene 31, 496778 (2012).

13. Pietrocola, F., Galluzzi, L., Bravo-San Pedro, J.M., Madeo, F. \& Kroemer, G. Acetyl coenzyme A: a central metabolite and second messenger. Cell Metab 21, 805-21 (2015).

14. Schug, Z.T. et al. Acetyl-CoA synthetase 2 promotes acetate utilization and maintains cancer cell growth under metabolic stress. Cancer Cell 27, 57-71 (2015).

15. Jeon, S.M., Chandel, N.S. \& Hay, N. AMPK regulates NADPH homeostasis to promote tumour cell survival during energy stress. Nature 485, 661-5 (2012).

16. Anastasiou, D. et al. Inhibition of pyruvate kinase $\mathrm{M} 2$ by reactive oxygen species contributes to cellular antioxidant responses. Science 334, 1278-83 (2011).

17. Sharpe, L.J. \& Brown, A.J. Controlling cholesterol synthesis beyond 3-hydroxy-3-methylglutarylCoA reductase (HMGCR). J Biol Chem 288, 18707-15 (2013).

18. Hart, T. et al. High-Resolution CRISPR Screens Reveal Fitness Genes and Genotype-Specific Cancer Liabilities. Cell 163, 1515-26 (2015).

MVA pathway genes were scored as essential across multiple cancer cell types, highlighting the dependency of cancer cells on the MVA pathway.

19. Wang, T. et al. Identification and characterization of essential genes in the human genome. Science 350, 1096-101 (2015).

20. Blomen, V.A. et al. Gene essentiality and synthetic lethality in haploid human cells. Science 350, 1092-6 (2015).

21. Clendening, J.W. et al. Exploiting the mevalonate pathway to distinguish statin-sensitive multiple myeloma. Blood 115, 4787-97 (2010). 
22. Goard, C.A. et al. Identifying molecular features that distinguish fluvastatin-sensitive breast

tumor cells. Breast Cancer Res Treat 143, 301-12 (2014).

23. Keyomarsi, K., Sandoval, L., Band, V. \& Pardee, A.B. Synchronization of tumor and normal cells from G1 to multiple cell cycles by lovastatin. Cancer Res 51, 3602-9 (1991).

24. Dimitroulakos, J. et al. Microarray and biochemical analysis of lovastatin-induced apoptosis of squamous cell carcinomas. Neoplasia 4, 337-46 (2002).

25. Dimitroulakos, J. et al. Increased sensitivity of acute myeloid leukemias to lovastatin-induced apoptosis: A potential therapeutic approach. Blood 93, 1308-18 (1999).

26. Larson, R.A. \& Yachnin, S. Mevalonic acid induces DNA synthesis in chronic lymphocytic leukemia cells. Blood 64, 257-62 (1984).

27. Clendening, J.W. et al. Dysregulation of the mevalonate pathway promotes transformation. Proc Natl Acad Sci U S A 107, 15051-6 (2010).

This study was the first to show that the rate-limiting enzyme of the MVA pathway, HMGCR, can promote transformation.

28. Duncan, R.E., El-Sohemy, A. \& Archer, M.C. Mevalonate promotes the growth of tumors derived from human cancer cells in vivo and stimulates proliferation in vitro with enhanced cyclindependent kinase-2 activity. J Biol Chem 279, 33079-84 (2004).

29. Bloch, K. The biological synthesis of cholesterol. Science 150, 19-28 (1965).

30. Goldstein, J.L. \& Brown, M.S. The low-density lipoprotein pathway and its relation to atherosclerosis. Annu Rev Biochem 46, 897-930 (1977).

31. Pike, L.J. The challenge of lipid rafts. J Lipid Res 50 Suppl, S323-8 (2009).

32. Mollinedo, F. \& Gajate, C. Lipid rafts as major platforms for signaling regulation in cancer. Adv Biol Regul 57, 130-46 (2015).

33. Ray, S., Kassan, A., Busija, A.R., Rangamani, P. \& Patel, H.H. The plasma membrane as a capacitor for energy and metabolism. Am J Physiol Cell Physiol, ajpcell 000872015 (2015).

34. York, A.G. et al. Limiting Cholesterol Biosynthetic Flux Spontaneously Engages Type I IFN Signaling. Cell 163, 1716-29 (2015).

35. Li, H.Y., Appelbaum, F.R., Willman, C.L., Zager, R.A. \& Banker, D.E. Cholesterol-modulating agents kill acute myeloid leukemia cells and sensitize them to therapeutics by blocking adaptive cholesterol responses. Blood 101, 3628-34 (2003).

36. Novak, A. et al. Cholesterol masks membrane glycosphingolipid tumor-associated antigens to reduce their immunodetection in human cancer biopsies. Glycobiology 23, 1230-9 (2013).

37. Ko, Y.J. \& Balk, S.P. Targeting steroid hormone receptor pathways in the treatment of hormone dependent cancers. Curr Pharm Biotechnol 5, 459-70 (2004).

38. Lin, C.Y. \& Gustafsson, J.A. Targeting liver $X$ receptors in cancer therapeutics. Nat Rev Cancer 15, 216-24 (2015).

39. Krycer, J.R. \& Brown, A.J. Cholesterol accumulation in prostate cancer: a classic observation from a modern perspective. Biochim Biophys Acta 1835, 219-29 (2013).

40. Miziorko, H.M. Enzymes of the mevalonate pathway of isoprenoid biosynthesis. Arch Biochem Biophys 505, 131-43 (2011).

41. Gruenbacher, G. \& Thurnher, M. Mevalonate metabolism in cancer. Cancer Lett 356, 192-6 (2015).

42. Thurnher, M. \& Gruenbacher, G. T lymphocyte regulation by mevalonate metabolism. Sci Signal 8, re4 (2015).

43. Meraviglia, S. et al. In vivo manipulation of Vgamma9Vdelta2 T cells with zoledronate and lowdose interleukin-2 for immunotherapy of advanced breast cancer patients. Clin Exp Immunol 161, 290-7 (2010). 
44. Dieli, F. et al. Targeting human \{gamma\}delta\} T cells with zoledronate and interleukin-2 for immunotherapy of hormone-refractory prostate cancer. Cancer Res 67, 7450-7 (2007).

45. Willumsen, B.M., Christensen, A., Hubbert, N.L., Papageorge, A.G. \& Lowy, D.R. The p21 ras Cterminus is required for transformation and membrane association. Nature 310, 583-6 (1984).

46. Hart, K.C. \& Donoghue, D.J. Derivatives of activated H-ras lacking C-terminal lipid modifications retain transforming ability if targeted to the correct subcellular location. Oncogene 14, 945-53 (1997).

47. Clarke, S., Vogel, J.P., Deschenes, R.J. \& Stock, J. Posttranslational modification of the Ha-ras oncogene protein: evidence for a third class of protein carboxyl methyltransferases. Proc Natl Acad Sci U S A 85, 4643-7 (1988).

48. Moores, S.L. et al. Sequence dependence of protein isoprenylation. J Biol Chem 266, 14603-10 (1991).

49. Casey, P.J. \& Seabra, M.C. Protein prenyltransferases. J Biol Chem 271, 5289-92 (1996).

50. Kang, S., Kim, E.S. \& Moon, A. Simvastatin and lovastatin inhibit breast cell invasion induced by H-Ras. Oncol Rep 21, 1317-22 (2009).

51. Pandyra, A. et al. Immediate utility of two approved agents to target both the metabolic mevalonate pathway and its restorative feedback loop. Cancer Res 74, 4772-82 (2014). This demonstrated the feasibility of targetting SREBP2 to potentiate the anti-cancer effects of statins.

52. Wong, W.W. et al. Determinants of sensitivity to lovastatin-induced apoptosis in multiple myeloma. Mol Cancer Ther 6, 1886-97 (2007).

This is one of the first studies to show that isoprenoids GGPP and FPP can reverse statininduced apoptosis.

53. Xia, Z. et al. Blocking protein geranylgeranylation is essential for lovastatin-induced apoptosis of human acute myeloid leukemia cells. Leukemia 15, 1398-407 (2001).

54. Agarwal, B. et al. Mechanism of lovastatin-induced apoptosis in intestinal epithelial cells. Carcinogenesis 23, 521-8 (2002).

55. Jiang, Z., Zheng, X., Lytle, R.A., Higashikubo, R. \& Rich, K.M. Lovastatin-induced up-regulation of the BH3-only protein, Bim, and cell death in glioblastoma cells. J Neurochem 89, 168-78 (2004).

56. Shellman, Y.G. et al. Lovastatin-induced apoptosis in human melanoma cell lines. Melanoma Res 15, 83-9 (2005).

57. Stirewalt, D.L., Appelbaum, F.R., Willman, C.L., Zager, R.A. \& Banker, D.E. Mevastatin can increase toxicity in primary AMLs exposed to standard therapeutic agents, but statin efficacy is not simply associated with ras hotspot mutations or overexpression. Leuk Res 27, 133-45 (2003).

58. Hentschel, A., Zahedi, R.P. \& Ahrends, R. Protein lipid modifications-More than just a greasy ballast. Proteomics 16, 759-82 (2016).

59. Berndt, N., Hamilton, A.D. \& Sebti, S.M. Targeting protein prenylation for cancer therapy. Nat Rev Cancer 11, 775-91 (2011).

This review comprehensively summarizes the feasibility and efficacy of targeting protein prenylation in cancer.

60. Cox, A.D., Der, C.J. \& Philips, M.R. Targeting RAS Membrane Association: Back to the Future for Anti-RAS Drug Discovery? Clin Cancer Res 21, 1819-27 (2015).

61. Cox, A.D., Fesik, S.W., Kimmelman, A.C., Luo, J. \& Der, C.J. Drugging the undruggable RAS: Mission possible? Nat Rev Drug Discov 13, 828-51 (2014).

62. Swanson, K.M. \& Hohl, R.J. Anti-cancer therapy: targeting the mevalonate pathway. Curr Cancer Drug Targets 6, 15-37 (2006).

63. Wiemer, A.J., Wiemer, D.F. \& Hohl, R.J. Geranylgeranyl diphosphate synthase: an emerging therapeutic target. Clin Pharmacol Ther 90, 804-12 (2011). 
64. Tsimberidou, A.M., Chandhasin, C. \& Kurzrock, R. Farnesyltransferase inhibitors: where are we now? Expert Opin Investig Drugs 19, 1569-80 (2010).

65. Martin, N.E. et al. A phase I trial of the dual farnesyltransferase and geranylgeranyltransferase inhibitor L-778,123 and radiotherapy for locally advanced pancreatic cancer. Clin Cancer Res 10, 5447-54 (2004).

66. Ullah, N., Mansha, M. \& Casey, P.J. Protein Geranylgeranyltransferase Type 1 as a Target in Cancer. Curr Cancer Drug Targets (2015).

67. Chojnacki, T. \& Dallner, G. The biological role of dolichol. Biochem J 251, 1-9 (1988).

68. Carlberg, M. et al. Mevalonic acid is limiting for $\mathrm{N}$-linked glycosylation and translocation of the insulin-like growth factor-1 receptor to the cell surface. Evidence for a new link between 3hydroxy-3-methylglutaryl-coenzyme a reductase and cell growth. J Biol Chem 271, 17453-62 (1996).

69. Pinho, S.S. \& Reis, C.A. Glycosylation in cancer: mechanisms and clinical implications. Nat Rev Cancer 15, 540-55 (2015).

This review summaries the role of aberrant glycosylation in cancer development and progression.

70. Cheng, C. et al. Glucose-Mediated N-glycosylation of SCAP Is Essential for SREBP-1 Activation and Tumor Growth. Cancer Cell 28, 569-81 (2015).

This study links glucose metabolism to the mevalonate pathway via N-glycosylation of SCAP.

71. Ernster, L. \& Dallner, G. Biochemical, physiological and medical aspects of ubiquinone function. Biochim Biophys Acta 1271, 195-204 (1995).

72. Maiuri, M.C. \& Kroemer, G. Essential role for oxidative phosphorylation in cancer progression. Cell Metab 21, 11-2 (2015).

73. Tan, A.S. et al. Mitochondrial genome acquisition restores respiratory function and tumorigenic potential of cancer cells without mitochondrial DNA. Cell Metab 21, 81-94 (2015).

74. Hua, X. et al. SREBP-2, a second basic-helix-loop-helix-leucine zipper protein that stimulates transcription by binding to a sterol regulatory element. Proc Natl Acad Sci U S A 90, 11603-7 (1993).

Brown and Goldstein follow up their Nobel-prize winning work by identifying SREBP2.

75. Yokoyama, C. et al. SREBP-1, a basic-helix-loop-helix-leucine zipper protein that controls transcription of the low density lipoprotein receptor gene. Cell 75, 187-97 (1993).

76. Seo, Y.K. et al. Genome-wide analysis of SREBP-1 binding in mouse liver chromatin reveals a preference for promoter proximal binding to a new motif. Proc Natl Acad Sci U S A 106, 13765-9 (2009).

77. Seo, Y.K. et al. Genome-wide localization of SREBP-2 in hepatic chromatin predicts a role in autophagy. Cell Metab 13, 367-75 (2011).

This study was the first to map the chromatin binding of SREBP2 genome-wide.

78. Fruman, D.A. \& Rommel, C. PI3K and cancer: lessons, challenges and opportunities. Nat Rev Drug Discov 13, 140-56 (2014).

79. Demoulin, J.B. et al. Platelet-derived growth factor stimulates membrane lipid synthesis through activation of phosphatidylinositol 3-kinase and sterol regulatory element-binding proteins. $J$ Biol Chem 279, 35392-402 (2004).

80. Zhou, R.H. et al. Vascular endothelial growth factor activation of sterol regulatory element binding protein: a potential role in angiogenesis. Circ Res 95, 471-8 (2004).

81. Fleischmann, M. \& Iynedjian, P.B. Regulation of sterol regulatory-element binding protein 1 gene expression in liver: role of insulin and protein kinase B/cAkt. Biochem J 349, 13-7 (2000).

82. Luu, W., Sharpe, L.J., Stevenson, J. \& Brown, A.J. Akt acutely activates the cholesterogenic transcription factor SREBP-2. Biochim Biophys Acta 1823, 458-64 (2012). 
83. Porstmann, T. et al. PKB/Akt induces transcription of enzymes involved in cholesterol and fatty acid biosynthesis via activation of SREBP. Oncogene 24, 6465-81 (2005).

84. Sundqvist, A. et al. Control of lipid metabolism by phosphorylation-dependent degradation of the SREBP family of transcription factors by SCF(Fbw7). Cell Metab 1, 379-91 (2005).

85. Yellaturu, C.R., Deng, X., Park, E.A., Raghow, R. \& Elam, M.B. Insulin enhances the biogenesis of nuclear sterol regulatory element-binding protein (SREBP)-1c by posttranscriptional downregulation of Insig-2A and its dissociation from SREBP cleavage-activating protein (SCAP).SREBP1c complex. J Biol Chem 284, 31726-34 (2009).

86. Hegarty, B.D. et al. Distinct roles of insulin and liver $X$ receptor in the induction and cleavage of sterol regulatory element-binding protein-1c. Proc Natl Acad Sci U S A 102, 791-6 (2005).

87. Yecies, J.L. et al. Akt stimulates hepatic SREBP1C and lipogenesis through parallel mTORC1dependent and independent pathways. Cell Metab 14, 21-32 (2011).

88. Du, X., Kristiana, I., Wong, J. \& Brown, A.J. Involvement of Akt in ER-to-Golgi transport of SCAP/SREBP: a link between a key cell proliferative pathway and membrane synthesis. Mol Biol Cell 17, 2735-45 (2006).

89. Yellaturu, C.R. et al. Insulin enhances post-translational processing of nascent SREBP-1c by promoting its phosphorylation and association with COPII vesicles. J Biol Chem 284, 7518-32 (2009).

90. Ricoult, S.J., Yecies, J.L., Ben-Sahra, I. \& Manning, B.D. Oncogenic PI3K and K-Ras stimulate de novo lipid synthesis through mTORC1 and SREBP. Oncogene (2015).

91. Yamauchi, Y., Furukawa, K. \& Hamamura, K. Positive feedback loop between PI3K-Akt-mTORC1 signaling and the lipogenic pathway boosts Akt signaling: induction of the lipogenic pathway by a melanoma antigen. Cancer Res 71, 4989-97 (2011).

92. Calvisi, D.F. et al. Increased lipogenesis, induced by AKT-mTORC1-RPS6 signaling, promotes development of human hepatocellular carcinoma. Gastroenterology 140, 1071-83 (2011).

93. Kusama, T. et al. 3-hydroxy-3-methylglutaryl-coenzyme a reductase inhibitors reduce human pancreatic cancer cell invasion and metastasis. Gastroenterology 122, 308-17 (2002).

94. Asslan, R. et al. Epidermal growth factor stimulates 3-hydroxy-3-methylglutaryl-coenzyme A reductase expression via the ErbB-2 pathway in human breast adenocarcinoma cells. Biochem Biophys Res Commun 260, 699-706 (1999).

95. DeBerardinis, R.J., Lum, J.J., Hatzivassiliou, G. \& Thompson, C.B. The biology of cancer: metabolic reprogramming fuels cell growth and proliferation. Cell Metab 7, 11-20 (2008).

96. Shimobayashi, M. \& Hall, M.N. Making new contacts: the mTOR network in metabolism and signalling crosstalk. Nat Rev Mol Cell Biol 15, 155-62 (2014).

97. Chung, J., Kuo, C.J., Crabtree, G.R. \& Blenis, J. Rapamycin-FKBP specifically blocks growthdependent activation of and signaling by the $70 \mathrm{kd}$ S6 protein kinases. Cell 69, 1227-36 (1992).

98. Kuo, C.J. et al. Rapamycin selectively inhibits interleukin-2 activation of p70 S6 kinase. Nature 358, 70-3 (1992).

99. von Manteuffel, S.R., Gingras, A.C., Ming, X.F., Sonenberg, N. \& Thomas, G. 4E-BP1 phosphorylation is mediated by the FRAP-p70s6k pathway and is independent of mitogenactivated protein kinase. Proc Natl Acad Sci U S A 93, 4076-80 (1996).

100. Porstmann, T. et al. SREBP activity is regulated by mTORC1 and contributes to Akt-dependent cell growth. Cell Metab 8, 224-36 (2008).

This study shows that activation of SREBPs through AKT-mTORC1 is required for cell growth.

101. Li, S., Brown, M.S. \& Goldstein, J.L. Bifurcation of insulin signaling pathway in rat liver: mTORC1 required for stimulation of lipogenesis, but not inhibition of gluconeogenesis. Proc Natl Acad Sci U S A 107, 3441-6 (2010). 
This study offers an explanation for the paradox of insulin resistance, where insulin fails to suppress glucose production but continues to promote lipid synthesis.

102. Duvel, K. et al. Activation of a metabolic gene regulatory network downstream of mTOR complex 1. Mol Cell 39, 171-83 (2010).

103. Wang, B.T. et al. The mammalian target of rapamycin regulates cholesterol biosynthetic gene expression and exhibits a rapamycin-resistant transcriptional profile. Proc Natl Acad Sci U S A 108, 15201-6 (2011).

104. Thoreen, C.C. et al. An ATP-competitive mammalian target of rapamycin inhibitor reveals rapamycin-resistant functions of mTORC1. J Biol Chem 284, 8023-32 (2009).

105. Liu, Q. et al. Development of ATP-competitive mTOR inhibitors. Methods Mol Biol 821, 447-60 (2012).

106. Peterson, T.R. et al. mTOR complex 1 regulates lipin 1 localization to control the SREBP pathway. Cell 146, 408-20 (2011).

New mTOR inhibitors enabled this work to identify a target of $m$ TOR that regulates SREBP activity.

107. Zhang, P., Verity, M.A. \& Reue, K. Lipin-1 regulates autophagy clearance and intersects with statin drug effects in skeletal muscle. Cell Metab 20, 267-79 (2014).

108. Shao, W. \& Espenshade, P.J. Expanding roles for SREBP in metabolism. Cell Metab 16, 414-9 (2012).

109. Griffiths, B. et al. Sterol regulatory element binding protein-dependent regulation of lipid synthesis supports cell survival and tumor growth. Cancer Metab 1, 3 (2013).

This is the first study to show that ablation of SREBPs impacts both lipid and protein biosynthesis.

110. Hardie, D.G. \& Alessi, D.R. LKB1 and AMPK and the cancer-metabolism link - ten years after. BMC Biol 11, 36 (2013).

111. Beg, Z.H., Allmann, D.W. \& Gibson, D.M. Modulation of 3-hydroxy-3-methylglutaryl coenzyme A reductase activity with cAMP and wth protein fractions of rat liver cytosol. Biochem Biophys Res Commun 54, 1362-9 (1973).

112. Beg, Z.H., Stonik, J.A. \& Brewer, H.B., Jr. 3-Hydroxy-3-methylglutaryl coenzyme A reductase: regulation of enzymatic activity by phosphorylation and dephosphorylation. Proc Natl Acad Sci U S A 75, 3678-82 (1978).

113. Clarke, P.R. \& Hardie, D.G. Regulation of HMG-CoA reductase: identification of the site phosphorylated by the AMP-activated protein kinase in vitro and in intact rat liver. EMBO $\mathbf{J}$, 2439-46 (1990).

114. Sato, R., Goldstein, J.L. \& Brown, M.S. Replacement of serine-871 of hamster 3-hydroxy-3methylglutaryl-CoA reductase prevents phosphorylation by AMP-activated kinase and blocks inhibition of sterol synthesis induced by ATP depletion. Proc Natl Acad Sci U S A 90, 9261-5 (1993).

115. Li, Y. et al. AMPK phosphorylates and inhibits SREBP activity to attenuate hepatic steatosis and atherosclerosis in diet-induced insulin-resistant mice. Cell Metab 13, 376-88 (2011).

116. Collins, S.P., Reoma, J.L., Gamm, D.M. \& Uhler, M.D. LKB1, a novel serine/threonine protein kinase and potential tumour suppressor, is phosphorylated by cAMP-dependent protein kinase (PKA) and prenylated in vivo. Biochem J 345 Pt 3, 673-80 (2000).

117. Houde, V.P. et al. Investigation of LKB1 Ser431 phosphorylation and Cys433 farnesylation using mouse knockin analysis reveals an unexpected role of prenylation in regulating AMPK activity. Biochem J 458, 41-56 (2014).

118. Freed-Pastor, W.A. et al. Mutant p53 disrupts mammary tissue architecture via the mevalonate pathway. Cell 148, 244-58 (2012). 
This study was the first to demonstrate that specific gain-of-function p53 mutants activate the mevalonate pathway in cancer cells.

119. Assaily, W. et al. ROS-mediated p53 induction of Lpin1 regulates fatty acid oxidation in response to nutritional stress. Mol Cell 44, 491-501 (2011).

120. Shamma, A. et al. Rb Regulates DNA damage response and cellular senescence through E2Fdependent suppression of N-ras isoprenylation. Cancer Cell 15, 255-69 (2009).

121. Dang, C.V. MYC on the path to cancer. Cell 149, 22-35 (2012).

122. Tu, W.B. et al. Myc and its interactors take shape. Biochim Biophys Acta 1849, 469-83 (2015).

123. Gao, P. et al. c-Myc suppression of miR-23a/b enhances mitochondrial glutaminase expression and glutamine metabolism. Nature 458, 762-5 (2009).

124. Meyer, N. \& Penn, L.Z. Reflecting on 25 years with MYC. Nat Rev Cancer 8, 976-90 (2008).

125. Wu, Y. et al. Srebp-1 Interacts with c-Myc to Enhance Somatic Cell Reprogramming. Stem Cells (2015).

126. An integrated encyclopedia of DNA elements in the human genome. Nature 489, 57-74 (2012).

127. Cao, Z. et al. MYC phosphorylation, activation, and tumorigenic potential in hepatocellular carcinoma are regulated by HMG-CoA reductase. Cancer Res 71, 2286-97 (2011).

128. Hofmann, J.W. et al. Reduced expression of MYC increases longevity and enhances healthspan. Cell 160, 477-88 (2015).

129. Sorrentino, G. et al. Metabolic control of YAP and TAZ by the mevalonate pathway. Nat Cell Biol 16, 357-66 (2014).

This provides compelling evidence of the importance of MVA pathway end-products in cancer.

130. Wang, Z. et al. Interplay of mevalonate and Hippo pathways regulates RHAMM transcription via YAP to modulate breast cancer cell motility. Proc Natl Acad Sci U S A 111, E89-98 (2014).

131. Mi, W. et al. Geranylgeranylation signals to the Hippo pathway for breast cancer cell proliferation and migration. Oncogene 34, 3095-106 (2015).

132. Aylon, Y. et al. The LATS2 tumor suppressor inhibits SREBP and suppresses hepatic cholesterol accumulation. Genes Dev 30, 786-97 (2016).

133. Riobo, N.A. Cholesterol and its derivatives in Sonic Hedgehog signaling and cancer. Curr Opin Pharmacol 12, 736-41 (2012).

134. Eaton, S. Multiple roles for lipids in the Hedgehog signalling pathway. Nat Rev Mol Cell Bio/9, 437-45 (2008).

135. Corcoran, R.B. \& Scott, M.P. Oxysterols stimulate Sonic hedgehog signal transduction and proliferation of medulloblastoma cells. Proc Natl Acad Sci U S A 103, 8408-13 (2006).

136. Nguyen, V.T. et al. Differential epigenetic reprogramming in response to specific endocrine therapies promotes cholesterol biosynthesis and cellular invasion. Nat Commun 6, 10044 (2015).

137. Locke, J.A. et al. Androgen levels increase by intratumoral de novo steroidogenesis during progression of castration-resistant prostate cancer. Cancer Res 68, 6407-15 (2008).

138. Ettinger, S.L. et al. Dysregulation of sterol response element-binding proteins and downstream effectors in prostate cancer during progression to androgen independence. Cancer Res 64, 221221 (2004).

139. Huang, W.C., Li, X., Liu, J., Lin, J. \& Chung, L.W. Activation of androgen receptor, lipogenesis, and oxidative stress converged by SREBP-1 is responsible for regulating growth and progression of prostate cancer cells. Mol Cancer Res 10, 133-42 (2012).

140. Goldstein, J.L. \& Brown, M.S. Regulation of the mevalonate pathway. Nature 343, 425-30 (1990).

141. Ahern, T.P. et al. Statin prescriptions and breast cancer recurrence risk: a Danish nationwide prospective cohort study. J Nat/ Cancer Inst 103, 1461-8 (2011). 
142. Fortuny, J. et al. Use of analgesics and nonsteroidal anti-inflammatory drugs, genetic predisposition, and bladder cancer risk in Spain. Cancer Epidemiol Biomarkers Prev 15, 1696-702 (2006).

143. Nielsen, S.F., Nordestgaard, B.G. \& Bojesen, S.E. Statin use and reduced cancer-related mortality. N Engl J Med 367, 1792-802 (2012).

\section{An impactful study showing reduced deaths from cancer in statin-users.}

144. Chae, Y.K. et al. Reduced risk of breast cancer recurrence in patients using ACE inhibitors, ARBs, and/or statins. Cancer Invest 29, 585-93 (2011).

145. Boudreau, D.M. et al. Comparative safety of cardiovascular medication use and breast cancer outcomes among women with early stage breast cancer. Breast Cancer Res Treat 144, 405-16 (2014).

146. Kwan, M.L., Habel, L.A., Flick, E.D., Quesenberry, C.P. \& Caan, B. Post-diagnosis statin use and breast cancer recurrence in a prospective cohort study of early stage breast cancer survivors. Breast Cancer Res Treat 109, 573-9 (2008).

147. Chen, C., Lin, J., Smolarek, T. \& Tremaine, L. P-glycoprotein has differential effects on the disposition of statin acid and lactone forms in mdr1a/b knockout and wild-type mice. Drug Metab Dispos 35, 1725-9 (2007).

148. Moon, H., Hill, M.M., Roberts, M.J., Gardiner, R.A. \& Brown, A.J. Statins: protectors or pretenders in prostate cancer? Trends Endocrinol Metab 25, 188-96 (2014).

149. Martirosyan, A., Clendening, J.C., Goard, C.A., Penn, L.Z. Lovastatin induces apoptosis of ovarian cancer cells and synergizes with doxorubicin: immediate therapeutic relevance. BMC Cancer In Press (2010).

150. Wong, W.W., Dimitroulakos, J., Minden, M.D. \& Penn, L.Z. HMG-CoA reductase inhibitors and the malignant cell: the statin family of drugs as triggers of tumor-specific apoptosis. Leukemia 16, 508-19 (2002).

151. Wong, W.W. et al. Cerivastatin triggers tumor-specific apoptosis with higher efficacy than lovastatin. Clin Cancer Res 7, 2067-75 (2001).

152. Dimitroulakos, J. et al. Lovastatin induces a pronounced differentiation response in acute myeloid leukemias. Leuk Lymphoma 40, 167-78 (2000).

153. Mas, E. \& Mori, T.A. Coenzyme Q(10) and statin myalgia: what is the evidence? Curr Atheroscler Rep 12, 407-13 (2010).

154. Harper, C.R. \& Jacobson, T.A. Evidence-based management of statin myopathy. Curr Atheroscler Rep 12, 322-30 (2010).

155. Bjarnadottir, O. et al. Targeting HMG-CoA reductase with statins in a window-of-opportunity breast cancer trial. Breast Cancer Res Treat 138, 499-508 (2013).

156. Garwood, E.R. et al. Fluvastatin reduces proliferation and increases apoptosis in women with high grade breast cancer. Breast Cancer Res Treat 119, 137-44 (2010).

The first window-of-opportunity, pre-operative trial to demonstrate that fluvastatin can reduce proliferation and increase apoptosis of tumour cells in women with high grade breast cancer.

157. Graf, H. et al. Chemoembolization combined with pravastatin improves survival in patients with hepatocellular carcinoma. Digestion 78, 34-8 (2008).

158. Kornblau, S.M. et al. Blockade of adaptive defensive changes in cholesterol uptake and synthesis in AML by the addition of pravastatin to idarubicin + high-dose Ara-C: a phase 1 study. Blood 109, 2999-3006 (2007).

159. Hus, M. et al. Thalidomide, dexamethasone and lovastatin with autologous stem cell transplantation as a salvage immunomodulatory therapy in patients with relapsed and refractory multiple myeloma. Ann Hematol 90, 1161-6 (2011). 
160. Jakobisiak, M. \& Golab, J. Statins can modulate effectiveness of antitumor therapeutic modalities. Med Res Rev 30, 102-35 (2010).

161. Shachaf, C.M. et al. Inhibition of HMGcoA reductase by atorvastatin prevents and reverses MYCinduced lymphomagenesis. Blood 110, 2674-84 (2007).

162. Hamilton, R.J. et al. Statin medication use and the risk of biochemical recurrence after radical prostatectomy: results from the Shared Equal Access Regional Cancer Hospital (SEARCH) Database. Cancer 116, 3389-98 (2010).

163. Harshman, L.C. et al. Statin Use at the Time of Initiation of Androgen Deprivation Therapy and Time to Progression in Patients With Hormone-Sensitive Prostate Cancer. JAMA Oncol 1, 495504 (2015).

164. Ho, Y.K., Smith, R.G., Brown, M.S. \& Goldstein, J.L. Low-density lipoprotein (LDL) receptor activity in human acute myelogenous leukemia cells. Blood 52, 1099-114 (1978).

165. Yue, S. et al. Cholesteryl ester accumulation induced by PTEN loss and PI3K/AKT activation underlies human prostate cancer aggressiveness. Cell Metab 19, 393-406 (2014).

166. Guillaumond, F. et al. Cholesterol uptake disruption, in association with chemotherapy, is a promising combined metabolic therapy for pancreatic adenocarcinoma. Proc Natl Acad Sci U S A 112, 2473-8 (2015).

167. Hirsch, H.A. et al. A transcriptional signature and common gene networks link cancer with lipid metabolism and diverse human diseases. Cancer Cell 17, 348-61 (2010).

168. Nazarian, R. et al. Melanomas acquire resistance to B-RAF(V600E) inhibition by RTK or N-RAS upregulation. Nature 468, 973-7 (2010).

169. Spagnolo, F., Ghiorzo, P. \& Queirolo, P. Overcoming resistance to BRAF inhibition in BRAFmutated metastatic melanoma. Oncotarget 5, 10206-21 (2014).

170. Pandyra, A.A. et al. Genome-wide RNAi analysis reveals that simultaneous inhibition of specific mevalonate pathway genes potentiates tumor cell death. Oncotarget 6, 26909-21 (2015).

171. Tuerdi, G. et al. Synergistic effect of combined treatment with gamma-tocotrienol and statin on human malignant mesothelioma cells. Cancer Lett 339, 116-27 (2013).

172. Krycer, J.R., Phan, L. \& Brown, A.J. A key regulator of cholesterol homoeostasis, SREBP-2, can be targeted in prostate cancer cells with natural products. Biochem J 446, 191-201 (2012).

This study highlights the potential of inhibiiting SREBP2 as an anti-cancer therapeutic.

173. Kamisuki, S. et al. A small molecule that blocks fat synthesis by inhibiting the activation of SREBP. Chem Biol 16, 882-92 (2009).

174. Li, X., Chen, Y.T., Hu, P. \& Huang, W.C. Fatostatin displays high antitumor activity in prostate cancer by blocking SREBP-regulated metabolic pathways and androgen receptor signaling. Mol Cancer Ther 13, 855-66 (2014).

175. Li, X., Wu, J.B., Chung, L.W. \& Huang, W.C. Anti-cancer efficacy of SREBP inhibitor, alone or in combination with docetaxel, in prostate cancer harboring p53 mutations. Oncotarget 6, 4101832 (2015).

176. Goldstein, J.L. \& Brown, M.S. A century of cholesterol and coronaries: from plaques to genes to statins. Cell 161, 161-72 (2015).

A retrospective of work uncovering and understanding the role of cholesterol in disease.

177. Saad, F. et al. A randomized, placebo-controlled trial of zoledronic acid in patients with hormone-refractory metastatic prostate carcinoma. J Natl Cancer Inst 94, 1458-68 (2002).

178. Aft, R. et al. Effect of zoledronic acid on disseminated tumour cells in women with locally advanced breast cancer: an open label, randomised, phase 2 trial. Lancet Oncol 11, 421-8 (2010). 
179. Morgan, G.J. et al. First-line treatment with zoledronic acid as compared with clodronic acid in multiple myeloma (MRC Myeloma IX): a randomised controlled trial. Lancet 376, 1989-99 (2010).

180. Harousseau, J.L. et al. A randomized phase 3 study of tipifarnib compared with best supportive care, including hydroxyurea, in the treatment of newly diagnosed acute myeloid leukemia in patients 70 years or older. Blood 114, 1166-73 (2009).

181. Sparano, J.A. et al. Phase II trial of tipifarnib plus neoadjuvant doxorubicin-cyclophosphamide in patients with clinical stage IIB-IIIC breast cancer. Clin Cancer Res 15, 2942-8 (2009).

182. Tang, J.J. et al. Inhibition of SREBP by a small molecule, betulin, improves hyperlipidemia and insulin resistance and reduces atherosclerotic plaques. Cell Metab 13, 44-56 (2011).

183. Guan, M. et al. Nelfinavir induces liposarcoma apoptosis through inhibition of regulated intramembrane proteolysis of SREBP-1 and ATF6. Clin Cancer Res 17, 1796-806 (2011).

184. Brunner, T.B. et al. Phase I trial of the human immunodeficiency virus protease inhibitor nelfinavir and chemoradiation for locally advanced pancreatic cancer. J Clin Oncol 26, 2699-706 (2008).

185. Rengan, R. et al. A phase I trial of the HIV protease inhibitor nelfinavir with concurrent chemoradiotherapy for unresectable stage IIIA/IIIB non-small cell lung cancer: a report of toxicities and clinical response. J Thorac Oncol 7, 709-15 (2012). 
Fig.1A. The mevalonate (MVA) pathway. The MVA pathway is an essential anabolic pathway that uses acetyl-CoA, derived from glucose, glutamine and/or acetate metabolism, to produce sterols and isoprenoid metabolites that are essential for a variety of biological processes. B. MVA pathway enzymes condense three acetyl-CoA molecules in a two-step reaction to produce 3-hydroxy-3-methylglutaryl coenzyme A (HMG-CoA). Both reactions are reversible and in equilibria, with the intracellular concentration of acetyl-CoA being the primary driver. HMG-CoA is then reduced by HMG-CoA reductase (HMGCR) to produce MVA via an irreversible reaction. MVA is then converted to isopentenyl diphosphate (IPP) through a series of enzymatic steps, which serves as a monomeric unit for the sequent synthesis of all downstream metabolites (highlighted in purple). Abbreviations: PPP $=$ pentose phosphate pathway, IDH = isocitrate dehydrogenase, ACAT2 = acetyl-CoA acetyltransferase 2, HMGCS1 = HMGCoA synthase 1, MVK = mevalonate kinase, $\mathrm{PMVK}=$ phosphomevalonate kinase, $\mathrm{MVD}=$ mevalonatediphosphate decarboxylase, IDI1/2 = isopentenyl diphosphate isomerase, FDPS = farnesyl diphosphate synthase, FDFT1 = farnesyl-diphosphate farnesyltransferase 1, GGPS1 = geranylgeranyl diphosphate synthase 1. Dashed lines indicate multiple steps.

Fig.2. The SREBP-regulated sterol feedback response controls the transcription of MVA pathway genes ${ }^{176}$. (i) When ER sterol concentrations are high, the full-length, precursor SREBPs are localized to the ER in a complex with SCAP and INSIG. This complex is maintained through the binding of sterols to SCAP and/or the binding of oxysterols to INSIG. (ii) When sterols are low, SCAP undergoes a conformational change that causes the SCAP/SREBP complex to dissociate from INSIG. SCAP is then able to bind COPII proteins and be transported in vesicles, with SREBP, to the Golgi. (iii) SREBP is sequentially cleaved by site-1 protease (S1P) and site-2 protease (S2P) at the Golgi. Although not indicated, S1P and S2P are transmembrane proteins (iv) The cleaved, mature SREBP can then translocate to the nucleus, where it homodimerizes and binds to sterol-response elements (SRE) in the promoter regions of its target genes to activate transcription.

Fig.3. SREBP processing and activity are regulated by PI3K signaling at multiple levels. (i, ii) AKT can increase SREBP expression and activity, in part via the inhibition of GSK3 $\beta$. (iii) mTORC1 increases SREBP processing and transcriptional activity through multiple substrates. mTORC1 activates S6K via phosphorylation to increase SREBP translocation, and potentially SREBP processing. (iv) The negative regulator of SREBP, LIPIN1, is also phosphorylated and inactivated by mTORC1. Despite the multiple levels of regulation of the SREBPs by PI3K signaling, the mechanisms remain to be elucidated and may be context-dependent.

Fig.4. Transcriptional control of MVA pathway gene transcription by oncogenes and tumour suppressors. (i) Specific gain-of-function p53 mutants functionally interact with SREBP to drive increased expression of MVA pathway genes. (ii) MYC can bind to SREBP to increase the expression of SREBP target genes and analysis of the ENCODE database shows that MYC and its binding partner, MAX, bind to the promoters of MVA pathway genes. (iii) The $\mathrm{pRB}$ tumour suppressor can interact with SREBP and reduce its binding at target genes. Loss of $\mathrm{pRB}$ in cancer removes this inhibition, leading to increased transcription of specific MVA pathway genes.

Fig.5. Activation of the MVA pathway drives oncogenic signaling pathways. (i) RhoA is required for the nuclear localization and activity of the YAP/TAZ oncogenes. The activity of RhoA is dependent on geranylgeranylation, which localizes RhoA to the plasma membrane. Geranylgeranylation requires GGPP produced exclusively via the MVA pathway, thus linking the MVA pathway to YAP/TAZ activity. (ii) Hedgehog $(\mathrm{Hh})$ signaling is involved in tumorigenesis in multiple cancer types, and $\mathrm{Hh}$ ligands require the covalent attachment of cholesterol for proper processing and activity. (iii) Cholesterol is the precursor for steroid hormones such as oestrogen and androgen. These hormones are involved in hormone-driven breast and prostate cancers. 
$1 \quad$ Fig.6. Inhibiting both the MVA pathway and the SREBP transcription factors is a viable cancer 2 therapeutic. Statins have potent anti-cancer properties. They inhibit HMGCR, thereby reducing MVA 3 pathway metabolites that are essential for cancer cell growth and survival (top panel). This triggers $4 \quad$ SREBP activation and transcription of MVA pathway genes, thus restoring MVA pathway activity 5 (bottom panel). This is a classic resistance mechanism and may explain why not all patients respond to 6 anti-cancer statin therapy. Dipyridamole is one example of an approved agent that inhibits SREBP

7 cleavage, preventing the restorative feedback response and increasing apoptosis in multiple cancer cells. 8 Combining these two approved drugs may increase the therapeutic response compared to statins alone. 
1 Table 1: Available agents, both experimental and clinically-approved, that target the MVA pathway, 2 production of its metabolites and/or its SREBP-regulated feedback mechanism.

\begin{tabular}{|c|c|c|c|c|}
\hline Drug class & & Target & $\begin{array}{l}\text { Stage of clinical } \\
\text { development }\end{array}$ & Refs \\
\hline \multirow[t]{2}{*}{$\begin{array}{l}\text { MVA } \\
\text { pathway } \\
\text { inhibitors }\end{array}$} & Statins & HMGCR & $\begin{array}{l}\text { FDA-approved as } \\
\text { cholesterol-lowering agents } \\
\text { and currently in phase I-III } \\
\text { clinical trials for the } \\
\text { treatment of various cancer } \\
\text { types }\end{array}$ & $155-159$ \\
\hline & Bisphosphonates & FDPS & $\begin{array}{l}\text { FDA-approved for the } \\
\text { treatment of osteoporosis, } \\
\text { patients with multiple } \\
\text { myeloma or solid tumour } \\
\text { bone metastases, in } \\
\text { combination with standard } \\
\text { therapy }\end{array}$ & $177-179$ \\
\hline $\begin{array}{l}\text { Prenylation } \\
\text { inhibitors }\end{array}$ & FTIs/GGTIs & $\begin{array}{l}\text { Farnesyl- and } \\
\text { geranylgeranyl } \\
\text {-transferases }\end{array}$ & $\begin{array}{l}\text { In phase I-III clinical trials } \\
\text { for the treatment of various } \\
\text { cancer types, as single } \\
\text { agents or in combination } \\
\text { with standard therapy }\end{array}$ & $\begin{array}{l}65,180, \\
181\end{array}$ \\
\hline \multirow{5}{*}{$\begin{array}{l}\text { SREBP } \\
\text { inhibitors }\end{array}$} & Fatostatin & SCAP & In pre-clinical development & 173-175 \\
\hline & Betulin & SCAP & In pre-clinical development & 182 \\
\hline & Tocotrienols & Unknown & In pre-clinical development & 171,172 \\
\hline & Nelfinavir & $\mathrm{S} 2 \mathrm{P}$ & $\begin{array}{l}\text { FDA-approved for the } \\
\text { treatment of HIV infection } \\
\text { and in phase I-II clinical } \\
\text { trials for the treatment of } \\
\text { various cancer types }\end{array}$ & $183-185$ \\
\hline & Dipyridamole & Unknown & $\begin{array}{l}\text { FDA-approved for the } \\
\text { prevention of cerebral } \\
\text { ischemia and in pre-clinical } \\
\text { development as an inhibitor } \\
\text { of SREBP }\end{array}$ & 51 \\
\hline
\end{tabular}

